and so cannot provide more than a very superficial coverage of the subject. There is, however, a list of references after most articles which serves as an introduction to sources which provide more detailed information. As might be expected, there is a considerable variation in the standard of the individual articles. Some provide an excellent thumb-nail sketch of the subject whereas others contrive to say very little indeed. The production quality is good and there are surprisingly few misprints for such a large book (although some inconsistencies have crept in, Abbe's name being rendered variously as Abbé or Abbee for example). The index is an essential feature of any reference book and in this case it is perfectly adequate.

\section{S. BRADbury}

\section{Raman analysis}

Applications of Laser Raman Spectroscopy. By Stanley K. Freeman. Pp. $x i+336$. (Wiley: New York and London, January 1974.) £9.75.

THIS new book on the applications of Raman spectroscopy is the most significant modern volume to survey the analytical value of laser Raman spectroscopy. It includes a compendium chapter on the principles behind the Raman and related effects and others on experimental technique, a long section on qualitative analytical applications and concludes with chapters on synthetic and biopolymers and the value of Raman methods in pollution studies.

Molecular vibrations are normally studied by infrared absorption and Raman scattering. Since the vibrations characteristic of a complex molecule are themselves complex, these two techniques can, in principle, provide excellent fingerprints of analytical value. Infrared spectroscopy has been used routinely in this way for more than thirty years but Raman spectroscopy has not. Forty years ago Raman spectroscopy was thought to have immense analytical potential but it became clear in the later 1930s that experimental limitations severely restricted the versatility of the method. The relatively recent advent of the laser and sophisticated commercial spectrometers have improved the situation dramatically with the result that Raman spectroscopy is now playing an invaluable part both as an analytical tool (in the broadest possible sense), in structural chemistry, solid state physics and many other diverse areas.

In his book, Freeman selects some of the analytical applications of Raman spectroscopy and discusses these in considerable detail. Since other books exist covering infrared and Raman spectra of crystals, and reviews abound in other detailed areas, he quite rightly spends most of his time on the area in which he is best known and which is worst surveyed elsewhere: the use of Raman spectroscopy for functional group identification in organic analysis. In this area, the book is unique and contains a wealth of otherwise unpublished data and the significant results of years of experience. In fact, it can be truthfully said to be the logical successor to the classic books in this field by Hibben and Kohlrausch, both published before 1940!

In the chapters on polymeric and biological compounds the coverage is again chemical and analytical rather than structural and little space is spent in considering the niceties of vibrational theory and structural consequences thereon. But in the context of the book as a whole, this is entirely relevant. The section on pollution monitoring and Remote Raman techniques is also valuable in that it surveys a field which is very diversely covered in the literature.

My initial reaction to Freeman's book was most unfavourable since the title is misleading. Nowhere convenient does the author point out that to him "applications" mean analytical ones, thus many will be disappointed by the narow coverage and extravagant claims on the dust cover. Further, the production quality is reminiscent of a cheap paperback. But once the first chapter is digested and the general tenor of the coverage grasped, the book endears itself to the practicing chemical vibrational spectroscopist.

\section{P. J. HENDRA}

\section{Aluminium analysed}

Analytical Chemistry of Aluminum. By V. N. Tikhonov. Translated by J. Schmorak. Pp. $x+303$. (Analytical Chemistry of the Elements.) (Halsted, Wiley: New York, Toronto and Chichester; Israel Program for Scientific Translations: Jerusalem, January 1974.) $£ 9.55$.

THIS latest volume in the series of monographs dealing with the analytical chemistry of the elements, and sponsored by the USSR Academy of Sciences through the Vernadskii Institute of Geochemistry and Analytical Chemistry, is concerned with aluminum (sic).

The contents follow the general pattern established in previous volumes. A short preliminary chapter details relevant information about the occurrence, the physical and chemical properties of aluminium and its compounds, particularly the properties of complexes of the element with organic compounds which have found use as analytical reagents. Chapter 2 contains an extensive account of chemical and physicochemical methods for the determination of aluminium, in which gravimetric, titrimetric, photometric and fluorimetric methods are given comprehensive coverage, rather less space being devoted to polarography, radioactivation and spectroscopic methods. Chapter 3 deals with the separation of aluminium from accompanying elements by precipitation with inorganic and organic reagents, by extraction, by a variety of chromotographic methods and by mercury cathode electrolysis. Chapter 4 treats the determination of aluminium in natural and industrial materials such as minerals, ores and industrial concentrates, soil, water and organic substances, metals and alloys. Chapter 5 brings the text to a conclusion with a description of methods for the determination of impurities in high-purity aluminium. The bibliography of work cited in the text contains over 1,300 entries. As with the earlier volumes in this series, the translation has been expertly done.

As the third most abundant element in the Earth's crust, aluminium is present in most natural and fabricated materials. Although its chemistry and hence its analytical chemistry is not particularly complicated, a vast literature on methods for its determination, particularly in the presence of interfering elements, now exists. Surprisingly, no attempt has been made to review this information since 1942, when Fischer contributed a monograph on the subject in the Handbook of Analytical Chemistry by Fresenius and Jander. The present text deals mainly with the literature up to 1968 with ocassional later references, none, of course, beyond 1971, the year of publication of the original Russian version, and covers all known methods for the determination of aluminium.

There can be no doubt about the value of this type of monograph to the practising analyst. Many of the problems associated with the analysis of aluminium-the ignition temperature of aluminium oxide, the $p \mathrm{H}$ for precipitation of aluminium 8-hydroxyuinolate, the difficulties of the complexometric titration, the volatilisation of aluminium for AAS, the best photometric reagent for a particular purpose -all are given thorough and realistic treatment. Although this book is essentially a review of existing literature, perhaps a major benefit is its presentation of Russian work not readily accessible to analytical chemists in the Western World. As a comprehensive and authoritative source of information on the analytical chemistry of aluminium, it must assume the premier role.
W. I. STEPHEN 